\title{
Systems of Care in Crisis: The Changing Nature of Palliative Care During COVID-19
}

\author{
Michael Chapman (D) • Beth Russell • Jennifer Philip
}

Received: 21 May 2020 / Accepted: 27 July 2020

(C) Journal of Bioethical Inquiry Pty Ltd. 2020

\begin{abstract}
Among the far-reaching impacts of COVID19 is its impact on care systems, the social and other systems that we rely in to maintain and provide care for those with "illness." This paper will examine these impacts through a description of the influence on palliative care systems that have arisen within this pandemic. It will explore the impact on the meaning of care, how care is performed and identified, and the responses of palliative care systems to these challenges. It will also highlight the current and potential future implications of these dynamics within the unfolding crisis of this pandemic.
\end{abstract}

\author{
M. Chapman \\ Department of Palliative Care, Canberra Hospital, ACT, Canberra, \\ Australia \\ M. Chapman \\ ANU Medical School, ACT, Canberra, Australia \\ M. Chapman $(\bowtie)$ \\ University of Technology Sydney, Sydney, NSW, Australia \\ e-mail: michael.chapman@anu.edu.au \\ B. Russell · J. Philip \\ Palliative Nexus, University of Melbourne and St Vincent's \\ Hospital Melbourne, Melbourne, Victoria, Australia \\ B. Russell · J. Philip \\ Department of Palliative Care, St Vincent's Hospital Melbourne, \\ Melbourne, Victoria, Australia \\ J. Philip \\ Victorian Comprehensive Cancer Centre, Melbourne, Victoria, \\ Australia
}

Keywords Bioethics · COVID-19 · Palliative care · Systems theory

We look towards each other no longer

From the old distance of our names;

Now you dwell inside the rhythm of breath,

As close to us as we are to ourselves.

(John O'Donohue, "On the death of the beloved")

Evident within the many lessons that the novel coronavirus (COVID-19) pandemic has taught us is the fragility of the systems that enable us to care for each other. Care systems, the dynamic interconnections and processes involving care providers and care recipients, result in the practices that we recognize as "care." These systems emerge from the needs of human bodies, the recognition and response to these needs by people and communities, the communication that supports these connections, and the meaning attributed to this care. These care systems are complex and develop through relationships and interactions (Hodiamont et al. 2019). We cannot understand them or predict their movement just by focusing on the individual parts (such as the people) involved (Glouberman and Zimmerman 2004). They are also adaptive. These care systems are influenced by and respond to the changes that occur within and around them, sometimes resulting in the emergence of new behaviours and approaches. Response can lead to some systems becoming more resilient to change and to other disrupting or even dispersing. How care is provided within and through these systems varies. But such systems remain an omnipresent shifting and often 
unnoticed tapestry of connections and structures engendering our experience (Maturana and Varela 1980).

As is true for so many of our current global experiences, it is no surprise that care systems have been affected by the threat or presence of COVID-19. COVID-19's primary direct impact is within the physical systems (the bodies) of infected people. However, this initial systemic, bodily impact of COVID-19 in individuals has consequences for other systems resulting in the social, psychological, and community impact of this crisis. These systemic changes also trigger responses from care systems, such as those providing palliative care.

\section{Palliative Care Systems}

Palliative care systems seek to perceive and respond to all the needs-physical, social, psychological, and spiritual-of the person with COVID-19 and their family as their central mission. In this pandemic it is notable that palliative care systems are more likely to be involved with older people or those with premorbid conditions for whom COVID-19 infection is more likely to result in severe illness or death (Zhou et al. 2020). Also relevant to palliative care systems has been the pandemic's profound influence on social, psychological, and community systems (particularly through uncertainty, isolation, and fear) which require responses and support. In particular, numerous reports of the feared reality of dying with COVID-19 in isolation exemplify the tragic challenge to our social and care systems (Wakam et al. 2020).

All "illness" induces responses from care systems. COVID-19, however, has also influenced the nature of these care systems' responses. The following discussion will focus upon the powerful challenge COVID-19 has presented for palliative care systems.

\section{The Meaning of Palliative Care}

The philosophical stance of palliative care systems and how these practices are understood by clinicians is challenged by COVID-19. Core to palliative care is the potential for healing and inner well-being, despite significant and progressive physical illness, and the importance of the clinicians' presence, attention, and receptivity to those they are caring for. These ideas are rooted in the ancient care philosophies of Asklepios, and their centrality to palliative care systems articulates relevant distinctions between palliative and other contemporary care practices (Randall and Downie 2006).

Awareness of these core, meaning-linked ideas assists us in identifying the impact of the need for physical distancing and personal protection on palliative care systems within the pandemic. Superficially, limiting the contact of staff who provide palliative care to the person with COVID-19 diminishes care system responses. More fundamentally, reducing "non-essential" contact with palliative care providers can stymie the development of therapeutic relationships with staff, diminishing the attention and presence which is definitional to these care practices. Additionally, isolating infected people from the people, places, and events that are meaningful to them and from non-clinician health-carers-such as social workers or spiritual care providers who are at particular risk of being determined as non-essentialcan limit the possibility of the healing and wellbeing within illness that is sought by palliative care. The consequences of COVID-19 for palliative care systems are not simply instrumental or related to notions of changes in care "quality" but fundamental to the central meaning of care.

COVID-19 has also influenced the meaning of being a palliative care provider. Palliative care clinicians' role satisfaction often corresponds to their sense that they are providing care which is beneficial and appreciated (Biagioli et al. 2018). However, the constraints encountered when delivering palliative care in the setting of COVID-19 may result in a compromised form of care that correspondingly influences the meaning of current care roles. Furthermore, new and relatively unfamiliar risks of being a palliative care provider have emerged which may be influential. Cancer or heart failure are not contagious, but healthcare providers are at increased risk of contracting the COVID-19 virus, particularly when a person is close to dying of COVID-19. Providing palliative care now places clinicians and their families at risk from the illness itself and from fear-based responses and hostility of the community to healthcare workers. 


\section{Connection, Communication, and Function}

Another challenge to palliative care systems arising from COVID-19 is through curtailing the participation of the community of those who require care in these care systems. In usual times the presence of families and friends at bedsides are critical supplements to palliative care while additionally themselves being recipients of care. The physical presence of families and friends is reassuring, supports our sense of place and meaning, and often has practical significance through their contribution to physical care acts. Families and friends enable palliative care systems through their presence and by speaking for the person when they are not able to, and in community settings may be major contributors to palliative care systems. Limiting the presence of families and friends diminishes these possibilities and in turn diminishes the impacts of care. As noted, families and friends are themselves also recipients of palliative attention as the community which cares for and is affected by the death of one of their own. Yet, enabling such care for social systems is complicated by the impacts of limited time and physical presence on building relationships of trust and shared understanding with care staff.

COVID-19 also influences the interactions through which care systems function, as both verbal and nonverbal communication are affected. The rapidity of the illness's transition from mild to severe may mean that key conversations have not taken place. The illness pattern of rapidly worsening symptoms and severe breathlessness impedes verbal communication (to or from the patient) when severe disease occurs. Additionally, the unfamiliar coverings of personal protective equipment obscure faces and facial expression, changing the manner and the comfort of the intimate interconnection associated with caregiving. Even a message written with or by a patient with COVID-19 is transformed from something valuable to something contagious.

Broader impacts on palliative care systems can also be recognized. The practice of palliative care requires an acceptance of uncertainty and the ability to convey assurance within turmoil. Part of this capacity arises from familiarity with the norms of death and dying and a deep awareness of what supports may be helpful and their availability. COVID-19 is an illness whose natural history and impact on the dying experience is only beginning to be understood (Kobayashi et al. 2020; Lovell et al. 2020). There is limited evidence to support potentially beneficial palliative care approaches, and the dependability and availability of usual supports and interventions has been undermined by concerns of scarcity, risk, and isolation. As a result, COVID-19 has challenged the confidence with which palliative care providers can reassure patients and earnestly commit to their usual core value of non-abandonment.

\section{Systemic Change and Transformation}

The consequences of COVID-19 have therefore influenced palliative care systems in multiple, developing ways. As noted, change in social systems is not an unusual or even necessarily problematic phenomenon, and even disruptive events can lead to responses which are transformative and ultimately beneficial. To some extent, ongoing reflective change maintains and defines these systems (Meadows 2008). But for all its necessity, change can also be destructive and disrupt or diminish the resilience of the systems that it affects. The threat that COVID-19 will induce wholesale disruption of healthcare systems generally, and palliative care systems specifically, is readily apparent. Reports of failures of palliative care provision arising from inadequate access to the necessary "stuff, staff, space or systems" required to provide this demonstrate the reality of these risks (Arya et al. 2020). In this context, such failures may negatively influence the daily experience, the grief, and the future expectations of caregivers and community members and perpetuate this pandemic's catastrophe for some communities for some time hence.

Some responses within palliative care systems to the pandemic represent an acceleration of changes already present or expected. The widespread adoption of IT solutions to supplement or replace physical presence and support healthcare provision provides evidence in practice. "Telehealth" has long been an available solution to transcend large physical distances and connect patients, families, and healthcare providers, but it has now become standard. The COVID-19 crisis and the availability of smart devices has meant that remote palliative care can transcend the critical spaces (sometimes across the space of a door) necessary to diminish the risk of infections. Further acceleration in remote connections from COVID-19 has been seen through an explosion of international palliative care collaborations as countries, services, and individuals seek to learn from other's experiences. A similar expansion in 
understanding the contribution of palliative care has also occurred as institutions seek to provide best care to all patients, including those patients who may benefit from treatment intensification and those who may not.

More fundamental responses have occurred across care and social systems with significant implications for the project of palliative care. The global threat has resulted in a broad and pervasive discussion about death and dying and acknowledgement of a need for support. Media attention has focused discussions of available resources upon acute and intensive care support (such as ICU bed spaces and ventilators) rather than the adequacy of palliative care systems. Perhaps this continued focus highlights and potentially amplifies the value that communities place on this highly technical medical model of dying to the detriment of different forms of care such as care by and for families, care at home, and care that acknowledges the normality of dying and prepares for it. Similarly, any attention afforded to palliative care has largely conceptualized this as an important alternative to "life-saving" care, undercutting decades of work exploring palliative care as meaning "good living" (and therefore appropriate to be considered early during significant illness) rather than only care for the dying. Aspects of our response to COVID19 even suggest that dying is becoming more invisible, hidden within the meaningless transparency of daily death-counts and not counted at all when it occurs outside an acute hospital (Spiegelhalter 2020).

\section{Emerging Implications}

While some of these immediate responses to change may seem useful, or at least understandable, their longer-term implications for care systems remains unclear. For instance, continued adoption of remote, ITdriven care may beneficially increase the accessibility of palliative care and result in desirable efficiencies actualized by a workforce and community now skilled and comfortable with these technological approaches. A greater focus on "remote" palliative care practices well beyond the period of the pandemic is likely, and desirable. Other potential implications for palliative care are less clear. For instance, it is foreseeable that these systemic movement toward remote communication might lead to a normativity of palliative care systems that are less intimate, less embodied, and more reliant on patients and families to stand in for the physical presence of clinicians. Such changes will doubtless lead to the emergence of new skills and capacities in clinicians. Whether these clinicians will be able to maintain the therapeutic quality of their presence and attention despite the physical separation implied by the need for lenses and screens and whether these previously core aspects of palliative care will have the same relevance remains to be seen. This is novel territory for palliative care systems filled with uncertainty and possibility.

Other longer-term implications may arise from these responses, and some have never seemed more necessary. Many hope that this pandemic will result in a better appreciation of the critical nature of palliative care to respond to the certain frailties of our human experience. The recognition that palliative care is an important, equal, and in some situations favourable alternative to resource-intensive care when resources are scarce AND also when resources are ample-rather than being equated with less or no care-is timely. Gross differences in global health equity persist despite ongoing attention, and global palliative care inequity is increasing (Knaul et al. 2015). The challenges of an ageing population, increasing chronic morbidity (Vos et al. 2015), the health pressures of the climate crisis (Friel 2019), and still future pandemics will underline the importance and necessity of palliative care provision for best care of our human populace.

In concluding, several points should be made. The commonality of responses of palliative care systems subject to the influences the COVID-19 pandemic noted with this work does not mean to imply a uniformity of experience. COVID-19 has had a heterogeneous impact on communities to date, and responses to the crisis at all levels are diverse including within palliative care systems. Given this complexity, we should be cautious in predicting what may result for these care systems. However, this diversity also implies that novel ideas and approaches to the problems that we face may be available to us elsewhere. Additionally, it is important to note that COVID-19 represents a change we are experiencing rather than a historic event viewed from afar. Successful spatial and social distancing has allowed many countries to "flatten the curve," but the implications for care systems of these new phases of our response and the mounting economic, political, and community interests in diminishing public health interventions are yet to be realized. It is necessary for us to recognize this uncertainty and the unfolding and adaptive influence it is having on care systems. Accepting the manifold and 
dynamic complexity of this period may allow further opportunities for us to respond to the crisis of COVID19 , for us to learn from the challenges of our experience to date, and to prepare our care systems, and ourselves, for what may come.

\section{References}

Arya, A., S. Buchman, B. Gagnon, and J. Downar. 2020. Pandemic palliative care: Beyond ventilators and saving lives. CMAJ 192(15): E400-E404.

Biagioli, V., C. Prandi, B. Nyatanga, and R. Fida. 2018. The role of professional competency in influencing job satisfaction and organizational citizenship behavior among palliative care nurses. Journal of Hospice and Palliative Nursing 20(4): 377-384.

Friel, S. 2019. Climate change and the people's health. New York: Oxford University Press.

Glouberman, S., and B. Zimmerman. 2004. Complicated and complex systems: What would successful reform of medicare look like? In Changing health care in Canada: The Romanow papers, vol. 2, edited by P-G. Forest, G. Marchildon, and T. McIntosh, 21-53. Toronto: University of Toronto Press.

Hodiamont, F., S. Jünger, R. Leidl, Bernd Oliver Maier, E. Schildmann, and C. Bausewein. 2019. Understanding complexity-The palliative care situation as a complex adaptive system. BMC Health Services Research 19(1): 1-14.

Knaul, F.M., P.E. Farmer, A. Bhadelia, P. Berman, and R. Horton. 2015. Closing the divide: The Harvard Global Equity Initiative-Lancet Commission on global access to pain control and palliative care. The Lancet 386(9995): 722-724.
Kobayashi, T., Sung-mok J., N.M. Linton, et al. 2020. Communicating the risk of death from novel coronavirus disease (COVID-19). Journal of Clinical Medicine 9(2): 580.

Lovell, N., M. Maddocks, S.N. Etkind, et al. 2020. Characteristics, symptom management and outcomes of 101 patients with COVID-19 referred for hospital palliative care. Journal of Pain and Symptom Management, 1-5.

Maturana, H., and F. Varela. 1980. Autopoesis and cognition: The realization of living. D. Reidel Publishing Company.

Meadows, D. 2008. Thinking in systems: A primer. White River Junction, VT: Chelsea Green Publishing.

Randall, F., and R.S. Downie. 2006. The philosophy of palliative care: Critique and reconstruction. Oxford: Oxford University Press.

Spiegelhalter, D. 2020. Coronavirus deaths: How does Britain compare with other countries? The Guardian, April 30. https://www.theguardian.com/commentisfree/2020/apr/30 /coronavirus-deaths-how-does-britain-compare-with-othercountries.

Vos, T., R.M. Barber, B. Bell, et al. 2015. Global, regional, and national incidence, prevalence, and years lived with disability for 301 acute and chronic diseases and injuries in 188 countries, 1990-2013: A systematic analysis for the global burden of disease study 2013. The Lancet 386 (9995): 743-800.

Wakam, G.K., J.R. Montgomery, B.E. Biesterveld, and C.S. Brown. 2020. Not dying alone-Modern compassionate care in the Covid-19 pandemic. New England Journal of Medicine 382: 1-2.

Zhou, F., T. Yu, R. Du, et al. 2020. Clinical course and risk factors for mortality of adult inpatients with COVID-19 in Wuhan, China: A retrospective cohort study. The Lancet 395(10229): 1054-1062.

Publisher's Note Springer Nature remains neutral with regard to jurisdictional claims in published maps and institutional affiliations. 